\title{
Airway Vascularity is not Associated with Airway Hyper-responsiveness in a Non-human Primate Model of Asthma
}

Mark Avdalovic ${ }^{*}$, Erica Weiss ${ }^{2}$, Charles Sylvia², Sherri Quesenberry², Nancy Tyler ${ }^{2}$, Lisa Miller ${ }^{2}$, Edward Schelegle ${ }^{2}$ and Dallas Hyde ${ }^{2}$

${ }^{1}$ Division of Pulmonary and Critical Care Medicine, UC Davis School of Medicine, USA

${ }^{2}$ California National Primate Research Center, UC Davis School of Veterinary Medicine, USA

\begin{abstract}
It is now accepted that the asthmatic airway is "remodeled" from its normal state and there is a clinical assumption that features of this remodeling are associated with airway hyper-responsiveness. One feature of airway remodeling is vascular remodeling where the airway has a higher density of vascularity than a normal airway. We used an established primate model of asthma to formally address the relationship between vascular remodeling, airway inflammation and hyper-responsiveness. Twenty-four male rhesus macaques were exposed to combination of filtered air, house dust mite allergen (HDMA) and ozone for six months. Airway hyper-responsiveness was measured at the end of the six-month exposure. Using a defined unbiased stereologic approach the density of airway blood vessels, epithelial expression of vascular endothelial growth factor (VEGF) and airway abundance of eosinophils was quantitated. The results demonstrated that combined ozone and HDMA exposure generated the highest degree of airway hyper-responsiveness. By contrast the HDMA only exposure generated the highest degree of airway VEGF expression, vascular density and number of eosinophils. Taken together these results imply that airway eosinophils, VEGF and increased vascular density are not the primary contributing factor to increased airway hyperresponsiveness in the rhesus macaque model of asthma.
\end{abstract}

Keywords: Airway hyper-responsiveness; House dust mite allergen; Asthma; Vascular endothelial growth factor

\section{Introduction}

The term airway remodeling is a collective term that describes the presumed simultaneous changes that are seen in chronic asthma. These pathologic features include 1) mucous cell hyperplasia 2) basement membrane thickening 3) airway smooth muscle hypertrophy and most recently added 4) vascular remodeling. Airway remodeling is frequently associated with a characteristic inflammation that includes eosinophils and Th2 lymphocytes but may also occur in absence of inflammation [1]. The significance of vascular remodeling has only recently been appreciated [2-5]. The first description of vascular remodeling was reported by Dunnill who demonstrated an increased prominence of the vascular bed in twenty patients who had died of acute asthma [6]. One of the presumed consequences of airway remodeling and vascular remodeling in particular is that the airway becomes hyper-reactive. In an effort to correlate vascular mediators with airway hyper-responsiveness and airway permeability Tseliou et al. recruited thirty eight subjects with severe refractory asthma (SRA), thirty five patient with stable moderate asthma and 20 controls and they quantitated the presence of several different vascular mediators from their induced sputum and found that there was a correlation between hyper-reactivity and an increase in vascular mediators [7]. Other researchers havefurther suggested the association between angiogenic factors and increased airway hyper-responsiveness [8-10]. One of the challenges in this field is that phenotypic changes in human asthma are difficult to study at the tissue level. Typically rodent models have been used to bridge our gap in our understanding of tissue level remodeling changes, however rodents lack the airway distribution and geometry that is seen in the human lung.

Our group has previously established that ozone exposure enhances the characteristic changes of airway remodeling that occur with allergen exposure in a rhesus monkey model of allergic asthma [11]. Monkeys exposed to a combination of ozone and allergen had increased airway hyper-reactivity, basement membrane remodeling and airway eosinophilia. We have also previously published that airway vascularity is increased with allergen exposure and is more significant at the distal airways5. With these and other studies we have highlighted the advantage of the rhesus macaque model, namely the ability to study a phenotypic change down to the airway level. The aims of the current study were to further examine the degree of vascular remodeling within the proximal and distal airways and attempt to associate regional changes in airway vascular remodeling with airway hyper-reactivity and airway eosinophilic inflammationby using archived tissue from a previously described cohort with demonstrated increase in airway hyper-responsiveness [11] (Figure 1).

\section{Methods}

\section{Animal and exposure protocol}

The study consisted of 24 male rhesus macaque monkeys (Macaca mulatta) were selected from the breeding colony at the California National Primate Research Center (CNPRC) and removed from their mothers at 2 days of age. The infants were placed in a nursery for bottlefeeding and 24-hour care. These infants were housed in a $4.2 \mathrm{~m}^{3}$ capacity exposure chambers with open temperature controlled incubators at the exposure facility at the CNPRC. Ventilation of chambers was cycled at a rate of 30 changes per hour with filtered air (FA). Three animals

*Corresponding author: Mark Avdalovic, UC Davis School of Medicine, Division of Pulmonary and Critical Care Medicine, 4150 V street PSSB \#3400, Davis, Ca. 95616, USA, Tel: 916-985-9300; E-mail: mark.avdalovic@ucdmc.ucdavis.edu

Received August 13, 2013; Accepted September 23, 2013; Published September 28, 2013

Citation: Avdalovic M, Weiss E, Sylvia C, Quesenberry S, Tyler N, et al. (2013) Airway Vascularity is not Associated with Airway Hyper-responsiveness in a Non-human Primate Model of Asthma. J Allergy Ther 4: 149. doi:10.4172/21556121.1000149

Copyright: @ 2013 Avdalovic M, et al. This is an open-access article distributed under the terms of the Creative Commons Attribution License, which permits unrestricted use, distribution, and reproduction in any medium, provided the original author and source are credited. 


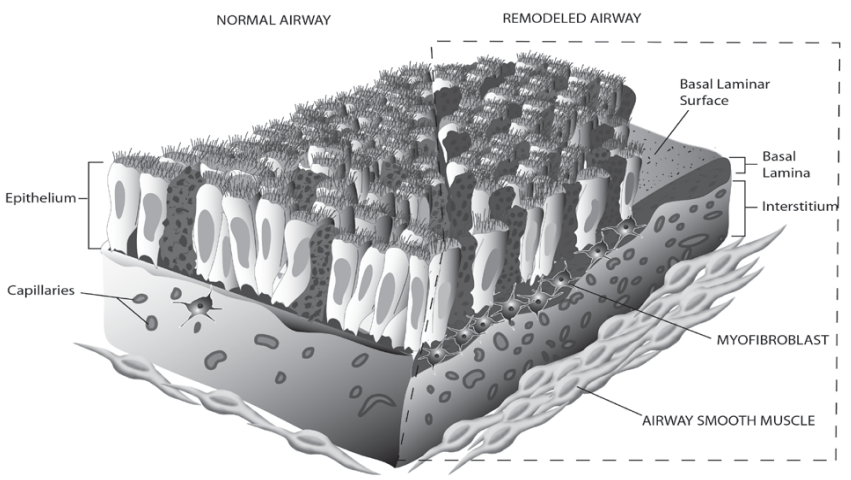

Figure 1: Airway Remodeling.

The schematic above is split figure with normal airway represented on the left and a remodeled asthmatic airway on the right. The key features of the remodeled airway include mucous cell hyperplasia, thickening of the basement membrane with associated proliferation of myofibroblast, increased vascular density and increased smooth muscle hypertrophy. Together these changes predispose to airway hyper-responsiveness.

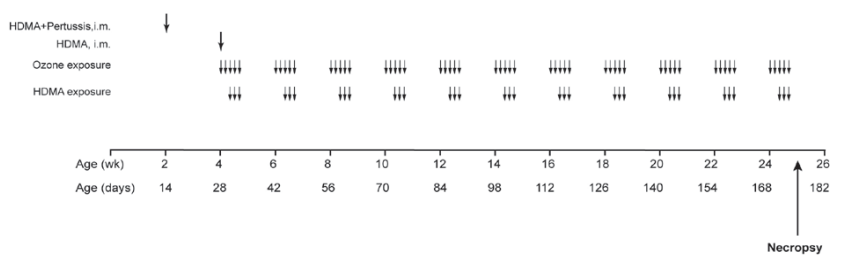

Figure 2: Exposure Diagram.

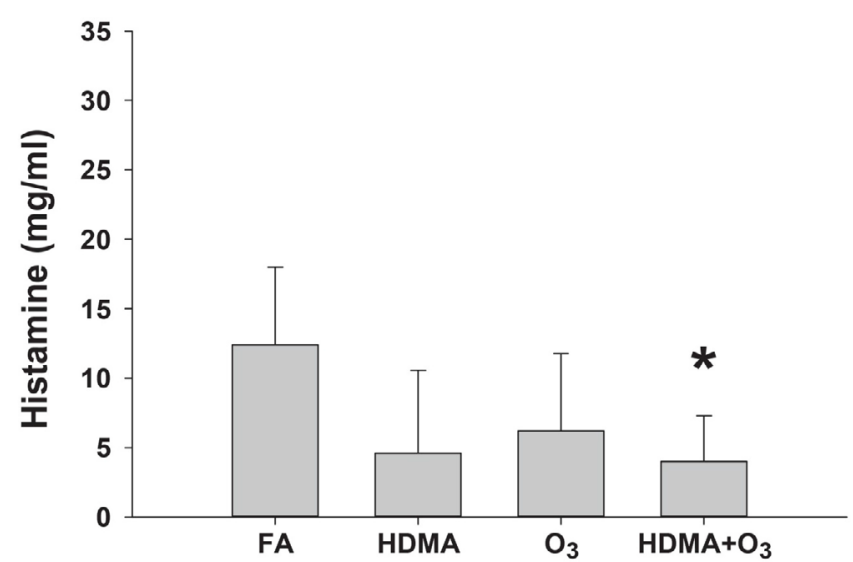

Figure 3: Airway Hyper-responsiveness

Airway hyper-reactivity is represented for each of the four exposure groups. The HDMA and ozone combined group had significantly more reactive airways compared to the filtered air group $\left({ }^{*} p=0.021\right)$.

were housed in each exposure chamber during the study. Experimental protocols were reviewed and approved by the University of California, Davis Institutional Animal Care and Use Committee. Care and housing of animals complied with the provisions of the Institute of Laboratory Animal resources and conformed to practices established by the Association for Assessment and Accreditation of Laboratory Animal Care (AAALAC). Animal studies conformed to applicable provisions of the Animal Welfare Act and other federal statutes and regulations relation to animals (Guide for the Care and Use of Laboratory Animals;
National Institutes of Health, revised 1985). The cohort of 24 male rhesus monkeys had exposure treatments for 6 months. Animals were randomly assigned to the following exposure groups within each cohort: (1) Filtered air control group, (2) aerosolized HDMA exposure, 3) Ozone exposed @ 0.5 ppm, (4) Ozone exposure (0.5 ppm) + HDMA exposure. At the end of 6 months these 24 monkeys went to necropsy. An outline of the exposure protocol is represented in Figure 2.

\section{Necropsy and immunohistochemistry}

After euthanasia, the entire mediastinal contents were removed en bloc. The lungs were then inflated with a $50 \% \mathrm{vol} / \mathrm{vol}$ mixture of optimal cutting temperature compound (OCT) and PBS. The tissue was then sliced perpendicular to the long axis of the main intrapulmonary conducting airway and frozen for cryosectioning. The tissue was then sectioned at $5 \mu \mathrm{m}$ at airway level 2 (bronchus level) and level 6 (bronchiole level). The immunohistochemistry protocol consisted of fixing the tissue for $10 \mathrm{~min}$ in acetone and allowed to air-dry for 30 minutes. The tissue was then re-hydrated and blocked with goat IgG $(20 \mu \mathrm{g} / \mathrm{mL}$ ). The slides were then incubated with the anti-CD31 (mouse anti-human) primary antibody, or anti VEGF (rabbit anti human) then washed with PBS. They were then incubated with the goat anti-mouse IgG or goat anti rabbit IgG (FITC) conjugated antibody, washed with PBS, coversliped with vectashield and read on a fluorescence microscope. A similar approach was taken with quantitation of eosinophils using an antibody to identify major basic protein (Biodesign Inc).

\section{Lung physiology}

Pulmonary mechanics were measured using a transfer impedance method as previously described [5]. All histamine challenges were administered as aerosols at a set maximal airway pressure $(10 \mathrm{~cm}$ $\mathrm{H}_{2} \mathrm{O}$ ) for a total of 30 breaths (1 breath/s) using a compressed air nebulizer (Vortran, Inc.) in series with a positive pressure ventilator (Bird Mark 7A respirator). Histamine challenges used repeated 30-s challenges separated by 240 -s data collection periods, beginning with saline followed by doubling concentrations starting at $0.0625 \mathrm{mg} / \mathrm{ml}$ and ending at the concentration that doubles Raw up to a maximum of $32 \mathrm{mg} / \mathrm{ml}$ or when $\mathrm{O}_{2}$ Sat\% dropped below $75 \%$. Data were expressed as the concentration that increases Raw by $150 \%$ (EC150Raw). The EC150Raw was determined by linear interpolation on the log-log plot of the dose-response curve with the response expressed as the percentage of baseline Raw.

\section{Stereology}

Intersection of vascular structures and basement membrane were counted using local vertical sections and a cycloid grid. Point counts of interstitium were used with intersections of the basement membrane to calculate surfaces per interstitial volume. Results are reported as surface area of vessels normalized to surface area of the basement membrane (Ss ves, bl). For VEGF stained tissue, point counts of VEGF positive events in epithelium were divided by total points of epithelium to obtain a volume density of VEGF in the epithelium. This value was then normalized by the surface area of the basement membrane per epithelial volume (Vs vegf,bl). Differences were considered significant when $\mathrm{P}<0.05$. All stereological measurements were obtained using Stereology Toolbox $^{\oplus}$ (Morpholmetrix). All data was normally distributed and analyzed by ANOVA (SYSTAT 10, SPSS., Chicago, Ill.). Vessel density, volume of epithelium, and volume of VEGF epithelial expression were determined for the four different treatment groups (Filtered air, HDMA, Ozone, and Ozone+HDMA) at two different airway levels, Levels 2 and 6, using previously described stereologic methods [12]. 


\section{Results}

\section{Airway hyper-responsiveness}

Airway hyper-responsiveness was measured at the end of the 6-month exposure period. Figure 3 shows the amount of histamine that was required to increase the baseline resistance for each monkey by $150 \%$. The less histamine that was required was reflective of a more hyper-reactive airway. There was a trend toward increased airway hyperresponsiveness for the HDMA and ozone groups but the HDMA and ozone combined group was the only group that was significantly more hyper-reactive compared with the filtered air control group $(\mathrm{p}=0.021)$. These results were previously published in 2003 [11].

\section{Vessel density (Ss ves, bl)}

Vessel density is represented as the ratio of the surface area of the

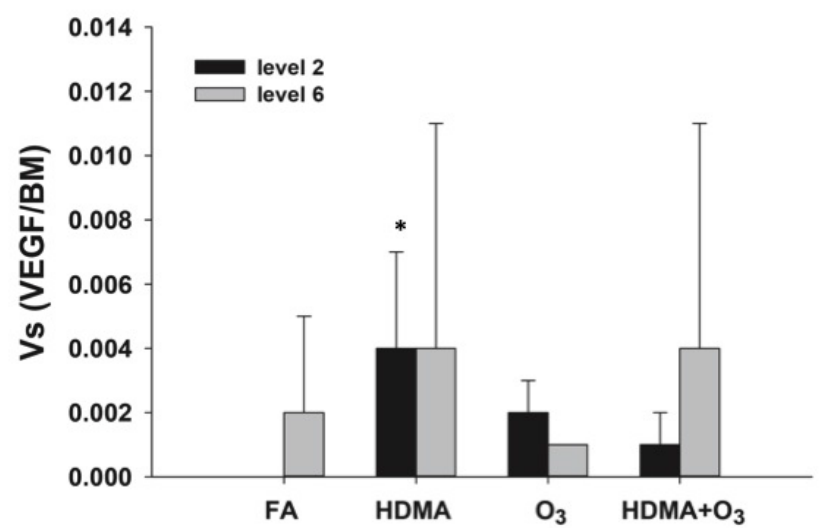

Figure 4: Vascular Surface Density (Ss Ves, BI)

The surface of airway blood vessel normalized to the surface of the basement membrane is represented for all four exposure groups at proximal (level 2) and distal (level 6) airways. At the distal airway level the HDMA group had a significantly higher vessel density compared to filtered air $\left(^{*}\right)$ and the two ozone groups had a significant decrease in vascular density compared to the HDMA group $\left({ }^{* *}\right)$.

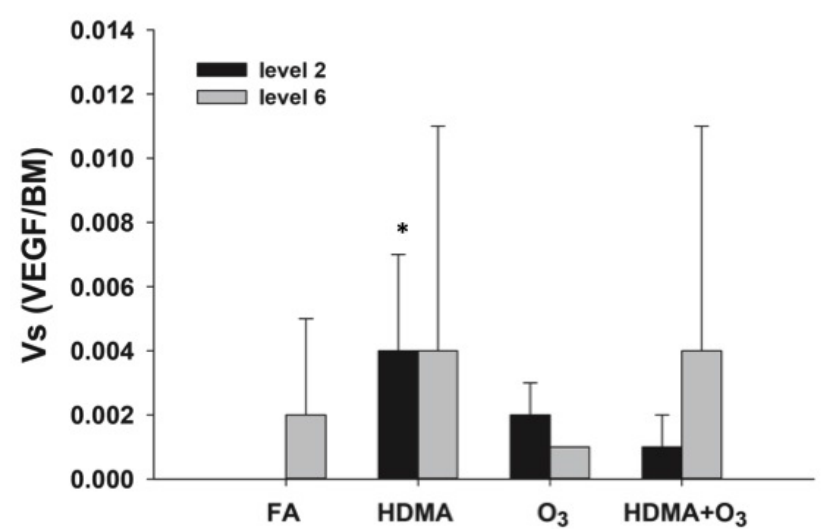

Figure 5: Volume of Epithelial VEGF (Vs VEGF, BI).

The volume of VEGF positive epithelial cells normalized to surface of basement membrane is represented for all four exposure groups at proximal (level 2) and distal (level 6) airway levels. The HDMA group had significant higher volume of epithelial VEGF expression compared to filtered air at the proximal airway level $\left({ }^{*} \mathrm{p}<0.05\right)$.

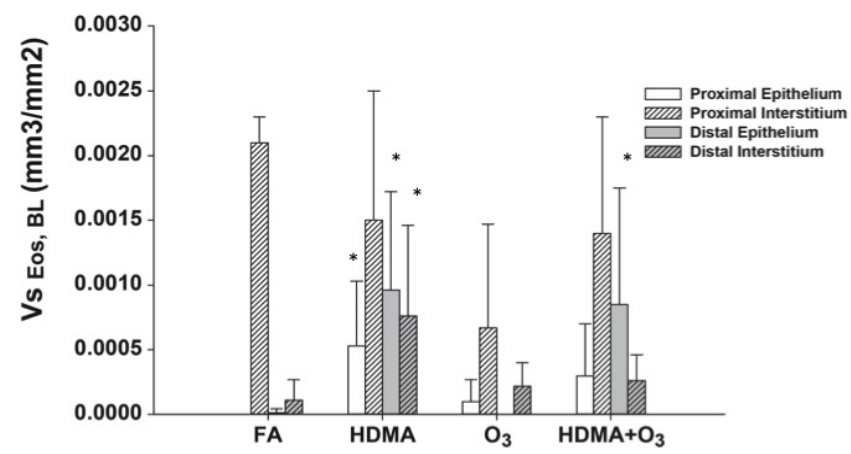

Figure 6: Volume of Eosinophils per Basement Membrane (Vs EOS,BL) The volume of eosinophils normalized to the surface of the basement membrane in the epithelial compartment and interstitium is represented. The volume of eosinophils was significantly greater in the proximal and distal epithelium as well distal interstitium for the HDMA group and distal epithelium for the HDMA/ Ozone group. ('signifies $p<0.05$ )

endothelium to the surface of epithelial basement membrane (Ss ves, bl) and is demonstrated in Figure 4. In comparison to the filtered air group, the vessel density in the HDMA exposure group was increased in both airway levels but only statistically significant in the level 6 airway $(p=0.02)$. Animals exposed to ozone+HDMA demonstrated a decrease in overall vessel density in both airway levels compared to HDMA but the difference was significant at airway level $6(\mathrm{p}=0.046)$. The ozone treated group showed a similar vessel density measurementto filtered air in airway level 2, but the vessel density decreasedin airway level 6 and was also significantly decreased compared to the HDMA group. The differences between the ozone and ozone+HDMA group were not statistically significant.

\section{Volume of epithelial VEGF per Epithelial basal lamina surface (Vs vegf, $b \mathbf{l}$ )}

The Vs vegf, bl (vegf volume per epithelial basal lamina surface) is illustrated in Figure 5. The HDMA exposure group had an approximately 9-fold statistically significant increase in VEGF per epithelial surface compared to filtered air in the level 2 airway $(p=0.02)$. Animals exposed to ozone+HDMA also had an overall increase in the Vs vegf, bl. The level 2 airway showed an approximately 4 -fold increase in the Vs vegf, $\mathrm{bl}$, which was statistically significant $(\mathrm{p}=0.051)$. In the level 6 airway, the Vs vegf, bl compared to the filtered air group and was not statistically significant. The ozone exposure group showed an overall increase in Vs vegf, bl in the level 2 airway, but the Vs vegf, bl decreased in the level 6 airway compared to the filtered air group.

\section{Airway inflammation}

The degree of eosinophilic airway inflammation is represented in Figure 6 as a volume fraction of eosinophils in the interstitial and epithelial compartments. The HDMA exposure group consistently had a significant increase in epithelial and interstitial eosinophils compared to the filtered air control. This increase was seen in the distal and proximal airways. By contrast the HDMA+ozone group had a significant increase in the distal epithelium but not in the other compartments. In previously reported research using this cohort of monkeys the bronchoalveolar lavage eosinophils were highest in the HDMA+ozone group [11]. 


\section{Correlation between airway remodeling and airway hyper- responsiveness}

Table 1 outlines the correlation between airway hyperresponsiveness and epithelial VEGF, airway vascularity and airway eosinophils. Correlation was first measured across the entire cohort (ungrouped) and was found not to be significant for airway vascular density, epithelial VEGF expression or airway eosinophils. When correlation was measured within each exposure group only the control group had a statistically significant $r$ value $(p<0.05)$, correlating epithelial VEGF density in the distal airway with hyper-responsiveness. This correlation became less significant for each of the exposure groups to the point of completely reversing the correlation in the HDMA/ ozone group.

Table 2 shows the correlation between epithelial VEGF expression and airway vascular density and airway eosinophils. Table 2 a shows the correlation for proximal airways while Table $2 \mathrm{~b}$ shows the correlation for the distal airways. Correlation was first examined across the entire cohort and then for each exposure group. The only significant correlation was a negative correlation between vessel density and distal epithelial VEGF expression in the combined HDMA/Ozone group. No significant positive correlation was identified between epithelial VEGF expression and measures of airway vascular density or airway eosinophilia.

\section{Discussion}

Vascular remodeling is a collective term to describe vascular changes in the airway in response to airway injury. Vascular remodeling has been associated as part of a multicellular response to airway injury, collectively termed airway remodeling. Early studies demonstrated that vascular remodeling is more prominent in airways of asthmatics [2].

\begin{tabular}{|c|c|c|c|c|c|}
\hline & UNGROUPED & Control & HDMA & Ozone & $\begin{array}{c}\text { HDMA/ } \\
\text { Ozone }\end{array}$ \\
\hline Vessel Density $_{\text {proximal airway }}$ & -0.08 & -0.42 & -0.38 & 0.28 & -0.465 \\
\hline Vessel Densitydistal airway & 0.01 & 0.19 & 0.02 & 0.01 & 0.33 \\
\hline Epithelial VEGF $_{\text {proximal airway }}$ & -0.13 & 0.37 & 0.42 & -0.27 & 0.401 \\
\hline Epithelial VEGF $_{\text {distal airway }}$ & -0.11 & $0.747^{*}$ & 0.25 & -0.02 & -0.610 \\
\hline Eosinophils $_{\text {EPP-proximal airway }}$ & -0.173 & 0 & -0.25 & -0.05 & -0.73 \\
\hline Eosinophils $_{\text {EPI-distal airway }}$ & -0.07 & -0.03 & -0.49 & 0 & 0.031 \\
\hline Eosinophils $_{\text {INT-proximal aimay }}$ & -0.08 & -0.49 & -0.04 & -0.55 & -0.64 \\
\hline Eosinophils $_{\text {INT-distal airway }}$ & -0.309 & -0.31 & 0.066 & -0.27 & -0.41 \\
\hline
\end{tabular}
$\mathrm{P}<0.05$

Table 1: Correlation with airway hyper-reactivity.

\begin{tabular}{|c|c|c|c|c|c|}
\hline & UNGROUPED & Control & HDMA & Ozone & $\begin{array}{c}\text { HDMA/ } \\
\text { Ozone }\end{array}$ \\
\hline Eosinophils Epithelium & -0.01 & 0 & -0.205 & -0.33 & -0.17 \\
\hline Eosinophils Interstitium & -0.05 & 0.18 & -0.3 & -0.57 & 0.03 \\
\hline Vessel Density & 0.07 & 0.67 & 0.530 & -0.48 & 0.420 \\
\hline
\end{tabular}

Table 2a: Correlation with epithelial VEGF-proximal airways.

\begin{tabular}{|c|c|c|c|c|c|}
\hline & UNGROUPED & Control & HDMA & Ozone & HDMA/Ozone \\
\hline $\begin{array}{c}\text { Eosinophils } \\
\text { Epithelium }\end{array}$ & 0.17 & -0.17 & -0.205 & 0 & 0.26 \\
\hline $\begin{array}{c}\text { Eosinophil } \\
\text { Interstitium }\end{array}$ & -0.03 & -0.37 & -0.3 & 0.03 & 0.04 \\
\hline $\begin{array}{c}\text { Vessel } \\
\text { Density }\end{array}$ & -0.05 & -0.19 & -0.41 & 0.045 & $-0.790^{*}$ \\
\hline
\end{tabular}

${ }^{*} \mathrm{P}<0.05$

Table 2b: Correlation with epithelial VEGF-distal airways.
There has even been evidence that patients with severe, therapy resistant asthma have an increase in vascular remodeling of the airways [13] Animal models, usually rodents, confirmed that vascular remodeling is associated with the airway remodeling that is characteristic of asthma. One animal model even demonstrated that VEGF over expression can increase vascular density and can also promote Th2 inflammation in the airway [14]. More recently researchers from the Severe Asthma Research Program reported that markers of vascular endothelial activation are associated with areas of air trapping on high resolution CT scans [15]. What virtually all of the studies have in common is that they implicate vascular remodeling as being a driving force or associated with a more severe asthma phenotype.

The rhesus macaque model of asthma allows for traditional clinical measure of asthma while also providing tissue to be analyzed at an airway specific level. We leveraged this valuable resource to study airway generation specific changes in vascular remodeling. Our previous work with this model had established that allergen exposure alone increases airway vascular density in both proximal and distal airways [5]. We used archived tissue from a previously reported cohort of 24-rhesus macaque randomized to four exposure conditions and measured airway vascular density and airway VEGF protein expression, which we associated with airway hyper-reactivity and airway eosinophils. The animal cohort that was used in this study combined ozone and HDMA exposure to generate a more asthmatic phenotype that included increased $\operatorname{IgE}$ levels, increased eosinophils on BAL and substantially increased airway hyper-responsiveness [11]. The principle aim was to measure airway vascular density and epithelial VEGF expression and correlates these changes with airway eosinophils and airway hyper-responsiveness.

Our findings demonstrated a decrease in airway vascularity when ozone is combined with HDMA exposure. These changes were most significant in the distal airways. Exposure with HDMA alone resulted in an increase in airway vascularity that was also most significant in the distal airway. By contrast, the expression of VEGF by airway epithelium was significantly increased with HDMA exposure in the proximal airway. We correlated the airway vascularity and epithelial VEGF expression with airway hyper-responsiveness and found no association of increased vascularity with increased hyper-responsiveness. In fact, the airways with the highest degree of increased vascularity and increased epithelial VEGF gene expression had some of the lower measurements of airway hyper-responsiveness. Moreover, the ozone combined with HDMA group had some of the lowest airway vascular density but some of the most reactive airways. Additionally the degree of airway eosinophilia also did not correlate with airway hyper-responsiveness and was most significantly increased in the HDMA exposure groups. Taken together, these results provide more granularities to the paradigm of increased airway remodeling leading to increased hyper-responsiveness.

This cohort of rhesus macaque had increased airway mucous cell hyperplasia, an irregular basement membrane and a loss in the overall volume in conducting airways was more significant in the combined exposure group of ozone and HDMA [11]. Although the airway eosinophils were not increased in the exposure group of ozone combined with HDMA, there was an increase in eosinophils on the bronchoalveolar lavage as well as serum IgE and histamine, implying that the combined exposure results in a more systemic allergic inflammatory response [11]. Could any of these changes contribute more to airway hyper-responsiveness than vascular remodeling changes? Certainly increased mucous cell hyperplasia may lead to airway obstruction which would affect airflow but also may lead toward volume loss and increase in airway resistance. Similarly a rise in 
Citation: Avdalovic M, Weiss E, Sylvia C, Quesenberry S, Tyler N, et al. (2013) Airway Vascularity is not Associated with Airway Hyper-responsiveness in a Non-human Primate Model of Asthma. J Allergy Ther 4: 149. doi:10.4172/2155-6121.1000149

circulating eosinophils in the pulmonary circulation could lead toward a decrease in lung capacity analogous to pulmonary edema. Finally, the combined exposure cohort had a decrease in the volume of conducting airways, reported in a previous publication [11]. This decrease alone could account for an increase in airway resistance and hyper-reactivity. Previous studies with the combined ozone and allergen model have highlighted the increased airway smooth muscle cell hypertrophy and increased density of intraepithelial nerves [16,17]. Both smooth muscle cell hypertrophy and increased density of intraepithelial airway nerves could also explain an increase airway hyper-responsiveness.

The current study however, is novel because it documents that advanced airway remodeling that results in an increase in airway hyper-responsiveness is not associated with increased airway vascular density or epithelial VEGF protein expression. This goes against previous reports that have established the presence of increased airway vascularity in patients with clinical asthma [18]. In fact, a recent study reported that airway hyper-reactivity was associated with increased sputum levels of sputum VEGF in patients with allergic rhinitis [19]. Using sputum VEGF as a biomarker of airway angiogenesis has never been validated and our current study is a more thorough evaluation of the contribution of airway vascular remodeling to airway physiology. Our study highlights the need to understand the full spectrum of beneficial as well as detrimental effects of increased vascular density in the asthmatic airway.

\section{References}

1. Grainge CL, Lau LC, Ward JA, Dulay V, Lahiff G, et al. (2011) Effect of bronchoconstriction on airway remodeling in asthma. N Engl J Med 364: 20062015.

2. Hashimoto M, Tanaka H, Abe S (2005) Quantitative analysis of bronchial wall vascularity in the medium and small airways of patients with asthma and COPD. Chest 127: 965-972.

3. Li X, Wilson JW (1997) Increased vascularity of the bronchial mucosa in mild asthma. Am J Respir Crit Care Med 156: 229-233.

4. Salvato G (2001) Quantitative and morphological analysis of the vascular bed in bronchial biopsy specimens from asthmatic and non-asthmatic subjects. Thorax 56: 902-906.

5. Avdalovic MV, Putney LF, Schelegle ES, Miller L, Usachenko JL, et al. (2006) Vascular remodeling is airway generation-specific in a primate model of chronic asthma. Am J Respir Crit Care Med 174: 1069-1076.

6. Dunnill MS (1960) The pathology of asthma, with special reference to changes in the bronchial mucosa. $\mathrm{J}$ Clin Pathol 13: 27-33.
7. Tseliou E, Bakakos P, Kostikas K, Hillas G, Mantzouranis K, et al. (2012) Increased levels of angiopoietins 1 and 2 in sputum supernatant in severe refractory asthma. Allergy 67: 396-402.

8. Papadaki G, Bakakos P, Kostikas K, Hillas G, Tsilogianni Z, et al. (2013) Vascular endothelial growth factor and cysteinyl leukotrienes in sputum supernatant of patients with asthma. Respir Med 107: 1339-1345

9. Hossny E, El-Awady H, Bakr S, Labib A (2009) Vascular endothelial growth factor overexpression in induced sputum of children with bronchial asthma. Pediatr Allergy Immunol 20: 89-96.

10. Abdel-Rahman AM, el-Sahrigy SA, Bakr SI (2006) A comparative study of two angiogenic factors: vascular endothelial growth factor and angiogenin in induced sputum from asthmatic children in acute attack. Chest 129: 266-271.

11. Schelegle ES, Miller LA, Gershwin LJ, Fanucchi MV, Van Winkle LS, et al. (2003) Repeated episodes of ozone inhalation amplifies the effects of allergen sensitization and inhalation on airway immune and structural development in Rhesus monkeys. Toxicol Appl Pharmacol 191: 74-85.

12. Hsia CC, Hyde DM, Ochs M, Weibel ER; ATS/ERS Joint Task Force on Quantitative Assessment of Lung Structure (2010) An official research policy statement of the American Thoracic Society/European Respiratory Society: standards for quantitative assessment of lung structure. Am J Respir Crit Care Med 181: 394-418

13. Vrugt B, Wilson S, Bron A, Holgate ST, Djukanovic R, et al. (2000) Bronchia angiogenesis in severe glucocorticoid-dependent asthma. Eur Respir J 15: 1014-1021.

14. Lee CG, Link H, Baluk P, Homer RJ, Chapoval S, et al. (2004) Vascular endothelial growth factor (VEGF) induces remodeling and enhances TH2mediated sensitization and inflammation in the lung. Nat Med 10: 1095-1103.

15. Johansson MW, Kruger SJ, Schiebler ML, Evans MD, Sorkness RL, et al (2013) Markers of vascular perturbation correlate with airway structural change in asthma. Am J Respir Crit Care Med 188: 167-178.

16. Plopper CG, Smiley-Jewell SM, Miller LA, Fanucchi MV, Evans MJ, et al. (2007) Asthma/allergic airways disease: does postnatal exposure to environmental toxicants promote airway pathobiology? Toxicol Pathol 35: 97-110.

17. Kajekar R, Pieczarka EM, Smiley-Jewell SM, Schelegle ES, Fanucchi MV, et al. (2007) Early postnatal exposure to allergen and ozone leads to hyperinnervation of the pulmonary epithelium. Respir Physiol Neurobiol 155: 55-63.

18. Hoshino M, Takahashi M, Aoike N (2001) Expression of vascular endothelia growth factor, basic fibroblast growth factor, and angiogenin immunoreactivity in asthmatic airways and its relationship to angiogenesis. J Allergy Clin Immunol 107: 295-301.

19. Yilmaz I, Bayraktar N, Ceyhan K, Secil D, Yuksel S, et al. (2013) Evaluation of vascular endothelial growth factor $A$ and endostatin levels in induced sputum and relationship to bronchial hyperreactivity in patients with seasonal allergic rhinitis. Am J Rhinol Allergy 27: 181-186. 